\title{
RAINBOW VERTEX-CONNECTION AND FORBIDDEN SUBGRAPHS
}

\author{
Wenjing Li, XUeliang Li ${ }^{1}$ \\ AND \\ JINGSHU ZHANG \\ Center for Combinatorics and LPMC \\ Nankai University \\ Tianjin 300071, China \\ e-mail: liwenjing610@mail.nankai.edu.cn \\ lxl@nankai.edu.cn \\ jszhang@mail.nankai.edu.cn
}

\begin{abstract}
A path in a vertex-colored graph is called vertex-rainbow if its internal vertices have pairwise distinct colors. A vertex-colored graph $G$ is rainbow vertex-connected if for any two distinct vertices of $G$, there is a vertexrainbow path connecting them. For a connected graph $G$, the rainbow vertexconnection number of $G$, denoted by $\operatorname{rvc}(G)$, is defined as the minimum number of colors that are required to make $G$ rainbow vertex-connected. In this paper, we find all the families $\mathcal{F}$ of connected graphs with $|\mathcal{F}| \in\{1,2\}$, for which there is a constant $k_{\mathcal{F}}$ such that, for every connected $\mathcal{F}$-free graph $G, \operatorname{rvc}(G) \leq \operatorname{diam}(G)+k_{\mathcal{F}}$, where $\operatorname{diam}(G)$ is the diameter of $G$.
\end{abstract}

Keywords: vertex-rainbow path, rainbow vertex-connection, forbidden subgraphs.

2010 Mathematics Subject Classification: 05C15, 05C35, 05C38, 05 C40.

\section{REFERENCES}

[1] G. Bacsó and Zs. Tuza, Dominating cliques in $P_{5}$-free graphs, Period. Math. Hungar. 21 (1990) 303-308. doi:10.1007/BF02352694

\footnotetext{
${ }^{1}$ Corresponding author.
} 
[2] J.A. Bondy and U.S.R. Murty, Graph Theory (GTM 244, Springer-Verlag, London, 2008).

[3] G. Chartrand, G.L. Johns, K.A. McKeon and P. Zhang, Rainbow connection in graphs, Math. Bohem. 133 (2008) 85-98.

[4] L. Chen, X. Li and Y. Shi, The complexity of determining the rainbow vertexconnection of a graph, Theoret. Comput. Sci. 412 (2011) 4531-4535. doi:10.1016/j.tcs.2011.04.032

[5] P. Holub, Z. Ryjáček, I. Schiermeyer and P. Vrána, Rainbow connection and foridden subgraphs, Discrete Math. 338 (2015) 1706-1713. doi:10.1016/j.disc.2014.08.008

[6] M. Krivelevich and R. Yuster, The rainbow connection of a graph is (at most) reciprocal to its minimum degree, J. Graph Theory 63 (2010) 185-191. doi:10.1002/jgt.20418

[7] S. Li, X. Li and Y. Shi, Note on the complexity of decidining the rainbow (vertex-) connectedness for bipartite graphs, Appl. Math. Comput. 258 (2015) 155-161. doi:10.1016/j.amc.2015.02.015

[8] X. Li and S. Liu, Tight upper bound of the rainbow vertex-connection number for 2-connected graphs, Discrete Appl. Math. 173 (2014) 62-69. doi:10.1016/j.dam.2014.04.002

[9] X. Li and Y. Shi, On the rainbow vertex-connection, Discuss. Math. Graph Theory 33 (2013) 307-313. doi:10.7151/dmgt.1664

[10] X. Li, Y. Shi and Y. Sun, Rainbow connections of graphs: A survey, Graphs Combin. 29 (2013) $1-38$. doi:10.1007/s00373-012-1243-2

[11] X. Li and Y. Sun, Rainbow Connections of Graphs (SpringerBriefs in Math., Springer-Verlag, New York, 2012).

Received 25 February 2016

Revised 21 October 2016

Accepted 21 October 2016 